\title{
Fluid Flow and Heat Transfer in Porous Metals Manufactured by Lost Carbonate Sintering
}

\author{
Yuyuan Zhao \\ School of Engineering, University of Liverpool \\ The Quadrangle, Brownlow Hill L69 3GH, \\ United Kingdom \\ Y.Y.Zhao@liverpool.ac.uk
}

Porous metals have high internal surface area and high permeability for fluids, which make them valuable material for thermal management applications, especially in heat dissipation by forced cooling. The high efficiency of heat transfer in porous metals is achieved by conduction through the metal matrix and convection by the coolant flowing through the interconnected pores. Fluid permeability and heat transfer coefficient of porous metals are two key parameters that are critical for practical thermal applications. Low permeability, or high flow resistance, means that high pumping power is required to move the working fluid through the cooling device. Heat transfer is also closely coupled to the fluid flow, which is strongly affected by the structure of the pore space. This presentation gives an overview of the fluid flow and heat transfer behaviour in porous copper manufactured by the Lost Carbonate Sintering (LCS) process. Experimental results on the permeability and heat transfer coefficient as a function of porosity, pore size and fluid flow rate are presented and discussed. 